\title{
Prevalence and Risk Factors of Elevated Liver Enzymes in Japanese Women With Polycystic Ovary Syndrome
}

\author{
Satomi Minato ${ }^{a}$, b, e , Naoki Sakane ${ }^{\mathrm{b}}$, Kazuhiko Kotani ${ }^{\mathrm{b}, \mathrm{c}}$, Shinsuke Nirengi ${ }^{\mathrm{b}}$, \\ Ikuyo Hayashia, b, Akiko Suganuma ${ }^{\text {b }}$, Ken Yamaguchid ${ }^{\text {, }}$ \\ Kenji Takakura ${ }^{\mathrm{d}}$, Narumi Nagai ${ }^{\mathrm{a}}$
}

\begin{abstract}
Background: Polycystic ovary syndrome (PCOS) is a common endocrine disorder among reproductive-aged women. While PCOS is associated with an increased risk of obesity and insulin resistance, little is known regarding the prevalence of and risk factors for nonalcoholic fatty liver disease (NAFLD) among Japanese women with PCOS. We estimated the prevalence of and risk factors for elevated liver enzymes, as the index of NAFLD, in Japanese women with PCOS.

Methods: We retrospectively reviewed 102 reproductive-aged women who visited the Department of Gynecology, Kyoto Medical Center in Japan from January 2000 to September 2016. Inclusion criterion was confirmed diagnosis of PCOS using International Classification of Diseases, 10th Revision, Clinical Modification (ICD-10) codes. Exclusion criteria were women with a history of liver diseases, missing body mass index (BMI) and serum alanine aminotransferase (ALT) data, and pregnancy. Data regarding age; BMI; and levels of blood glucose, serum lipid, liver enzymes, and sex hormones were obtained from medical records. Elevated liver enzymes was defined as ALT > $19 \mathrm{IU} / \mathrm{L}$. Optimal cutoffs for risk factors for elevated liver enzymes were calculated to determine predictors of elevated liver enzymes using area under the curve (AUC) by receiver-operating characteristics (ROC).
\end{abstract}

Results: The prevalence of elevated liver enzymes was 33.3\%. BMI was significantly higher in PCOS patients than in those without elevated liver enzymes ( 25.3 vs. $\left.20.7 \mathrm{~kg} / \mathrm{m}^{2}, \mathrm{P}<0.05\right)$. ROC analyses were performed using BMI and blood glucose and testosterone lev-

Manuscript submitted September 27, 2018, accepted October 15, 2018

${ }^{a}$ Graduate School of Human Science and Environment, University of Hyogo, Hyogo, Japan

${ }^{b}$ Division of Preventive Medicine, Clinical Research Institute, National Hospital Organization Kyoto Medical Center, Kyoto, Japan

${ }^{\mathrm{c} D i v i s i o n}$ of Community and Family Medicine, Jichi Medical University, Tochigi, Japan

dDepartment of Obstetrics and Gynecology, National Hospital Organization Kyoto Medical Center, Kyoto, Japan

'Corresponding Author: Satomi Minato, University of Hyogo, 1-1-12 Shinzaike-honcho, Himeji, Hyogo, 670-0092, Japan.

Email: minatosatomi@gmail.com

doi: https://doi.org/10.14740/jocmr3639 els because BMI and blood glucose showed differences between the groups and testosterone is related to fatty liver. AUC of the model including BMI and blood glucose and testosterone levels was 0.861 (sensitivity, 66.7\%; specificity, 100\%).

Conclusions: These findings suggest that elevated liver enzymes are common in women with PCOS. An algorism using BMI and blood glucose and testosterone levels might be useful to determine elevated liver enzymes in women with PCOS. Our finding may be useful for the study of NAFLD among Japanese women with PCOS since several previous studies have indicated elevated liver enzymes to be related to the potential presence of NAFLD. Further examination, including abdominal ultrasonography and/or liver biopsy data, is required to confirm these results.

Keywords: Polycystic ovary syndrome; Alanine aminotransferase; Obesity; Impaired glucose tolerance; Nonalcoholic fatty liver disease; Japanese

\section{Introduction}

Nonalcoholic fatty liver disease (NAFLD) is a hepatic manifestation of metabolic syndrome [1], and its pathology ranges from simple steatosis to cirrhosis and hepatocellular carcinoma without excessive alcohol intake [2]. The global prevalence of NAFLD in the general population is $25 \%$, and NAFLD incidence rate in Asia is 52 per 1,000 person-years [3]. Approximately half of NAFLD patients are obese and $23 \%$ have diabetes [3]. Elevated liver enzyme is one of the main characteristics of NAFLD. Moreover, age and menopause are important risk factors for NAFLD. Although $6 \%$ of the Japanese premenopausal women have NAFLD [4], etiology of NAFLD in premenopausal women remains unclear.

Meanwhile, polycystic ovary syndrome (PCOS) is the most common endocrine disease in reproductive-aged women [5], which is characterized by hyperandrogenism, chronic anovulation, and infertility [6]. The prevalence of PCOS is estimated at $8.0 \%$ in black and $4.8 \%$ in white premenopausal women [5]. More than $40 \%$ of women with PCOS have insulin resistance [7] and may have an increased prevalence of hypertension, dyslipidemia, diabetes and obesity [8].

NAFLD frequently occurs in women with PCOS. A recent 
meta-analysis has reported that women with PCOS have an approximately four-fold increased risk of NAFLD [9]. The prevalence of NAFLD in women with PCOS may vary worldwide because of their different genetic, ethnic, and lifestyle backgrounds. However, NAFLD prevalence in Japanese women with PCOS remains unclear. In addition, the identification of risk factors for NAFLD in PCOS is required to provide clinical references for early diagnosis and prevention. Several clinical measurements, such as age, body mass index (BMI), waist-tohip ratio, obesity, triglyceride level, homeostatic model assessment for insulin resistance (HOMA-IR) index, and androgen level, are considered to be associated with NAFLD in women with PCOS [10, 11].

Recently, several studies have applied alanine aminotransferase (ALT) measurement as a surrogate approach for the diagnosis of NAFLD instead of liver biopsy or ultrasonography [12-14]. In a previous cross-sectional Japanese study, ALT $=17$ IU/L was set as a cutoff for female NAFLD [15]. Furthermore, ALT $>19 \mathrm{IU} / \mathrm{L}$ was associated with an eight times increased mortality rate due to liver diseases. Importantly, the American College of Gastroenterology has recently proposed the upper limit of normal (ULN) for ALT to be 19 - 25 IU/L for females [16]. Currently, neither measurements of liver enzymes, including ALT, nor ultrasonography of the liver are performed in daily practice and for the diagnosis of PCOS. In addition, for screening and early detection of NAFLD in reproductiveaged women, it is useful to determine these predictors based on general examination indexes used in daily practice. Thus, we estimated the prevalence of and identified the risk factors for NAFLD using elevated liver enzymes and general examination indexes in Japanese women with PCOS.

\section{Materials and Methods}

\section{Subjects and data collection}

Study subjects were enrolled by reviewing electronic medical records between January 2000 and September 2016 from the National Hospital Organization Kyoto Medical Center in Japan. Subjects visited the Department of Gynecology and underwent physical examination and laboratory blood tests as part of the clinical workup. The Japanese Society for Obstetrics and Gynecology criteria [17, 18] were used. PCOS was diagnosed according to three main characteristics: 1) Cycle irregularities; 2) Polycystic ovaries on ultrasonography; and 3) Endocrine anomalies (high levels of luteinizing hormone (LH) or androgen). A high LH level was defined as $\mathrm{LH} \geq 7 \mathrm{IU} / \mathrm{L}$ and $\mathrm{LH} \geq$ follicle stimulating hormone (FSH) level in subjects with $\mathrm{BMI}<25 \mathrm{~kg} / \mathrm{m}^{2}$, and $\mathrm{LH} \geq \mathrm{FSH}$ in those with BMI $\geq 25 \mathrm{~kg} /$ $\mathrm{m}^{2}$ [19].

To identify women with PCOS, the International Classification of Diseases, 10th Revision, Clinical Modification (ICD-10) codes were used. Inclusion criterion was confirmed diagnosis of PCOS using ICD-10 codes for E282. Subjects were excluded if they had a history of liver diseases, missing BMI and ALT data, or pregnancy. ICD-10 codes were used especially for liver diseases, such as acute viral hepatitis
(B199), hemochromatosis (E831), and autoimmune liver disease (K754). In addition, to identify alcohol drinkers, ICD-10 codes from F100 to F107 (alcohol-related diseases) were used. A database of BMI and levels of serum liver enzymes, lipids, blood glucose, and sex hormones (FSH, LH, prolactin, estradiol and testosterone) was constructed on the basis of medical records; neutrophil-to-lymphocyte (NLR) and platelet-to-lymphocyte (PLR) ratios were used as inflammatory markers [20]. This study was conducted in accordance with the recommendation of principles of the Declaration of Helsinki (Fortaleza 2003) and was approved by the Ethics Committee of National Hospital Organization Kyoto Medical Center (approval number: 18-015). Opt-out informed consent was obtained from all subjects.

\section{Noninvasive NAFLD estimated index}

Clinical diagnosis and assessment of NAFLD by liver biopsy is regarded as the gold standard, but liver biopsy is not appropriate for the screening of NAFLD in reproductive-aged women owing to invasiveness [10] and sampling errors [21]. Moreover, its invasiveness may result in potential complications. In addition, the diagnostic accuracy of ultrasonography depends on the operator experience and skill [22], and these data were not available in this retrospective large-scale cohort study. Therefore, we considered that for screening and early detection of NAFLD in reproductive-aged women with PCOS, it is useful to determine the predictors based on general examination indexes used in daily practice.

The most common clinical indexes in NAFLD are elevated liver enzymes. Among liver enzymes, ALT is most closely related to liver fat accumulation [23] and is especially useful as a noninvasive independent predictor of incident NAFLD [24]. Recently, abnormal serum ALT levels have been regarded as indicative of NAFLD in reproductive-aged women [12-15]. Prati et al [12] have updated the upper limit of healthy range ALT to $>19 \mathrm{IU} / \mathrm{L}$ in women, and the American College of Gastroenterology has proposed 19 - 25 IU/L as female ULN of ALT [16]. In addition, Targher et al [13] have used ALT > $19 \mathrm{IU} / \mathrm{L}$ to define NAFLD in Italian women. Therefore, we defined ALT > $19 \mathrm{IU} / \mathrm{L}$ as elevated liver enzymes and regarded it as NAFLD estimated index. To study the prevalence and optimal cutoffs for risk factors for elevated liver enzymes, the study subjects were divided into two groups: women with PCOS alone (control group) and women with PCOS and ALT $>19$ IU/L (elevated liver enzymes group).

\section{Statistical analysis}

Continuous variables are presented as median (25 - 75 percentiles). The normality distribution of the variables was tested using the Kolmogorov-Smirnov test. Differences between the two groups (elevated liver enzymes vs. control groups) were compared with Student's $t$-test for normally distributed data and the Mann-Whitney U-test for nonnormally distributed data. A Spearman's correlation analysis was performed. BMI was significantly different $(\mathrm{P}<0.01)$, and blood glucose 


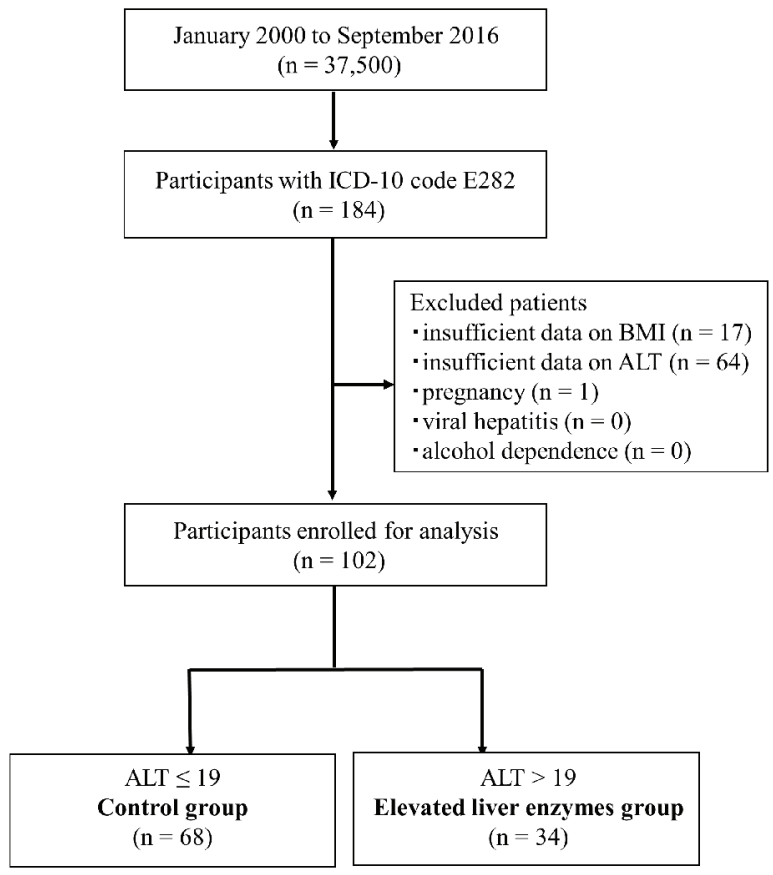

Figure 1. Inclusion and exclusion flow chart. Flow chart representing the selection of subjects during the retrospective study. Data of 37,500 Japanese women were reviewed, and 184 reproductive-aged women with PCOS were identified using ICD-10 codes. After excluding 82 women (see Materials and Methods section), the remaining subjects were divided into two groups using cutoffs for ALT (19 IU/L). ICD-10: International Classification of Diseased, 10th revision, clinical modification; ALT: alanine amino transferase.

showed a tendency to be different $(\mathrm{P}=0.07)$ between the two groups. BMI, blood glucose, and testosterone are related to fatty liver $[10,11,25]$. Therefore, we set up two models for predicting elevated liver enzymes using receiver-operating characteristics (ROC) curves to determine clinical references for early diagnosis and prevention. Model 1 included BMI and blood glucose levels, and Model 2 included BMI and levels of blood glucose and testosterone. The area under ROC curve (AUC) was calculated to assess overall diagnostic accuracy and to identify optimal cutoffs for risk factors for elevated liver enzymes. AUC was assessed using standard criteria [26] and interpreted as follows: excellent $(\geq 0.90)$, good $(0.80-0.89)$, fair $(0.70-0.79)$, or poor $(<0.70)$. The optimal cutoffs were determined, and the sensitivity, specificity, positive (PPV) and negative (NPV) predictive values, and diagnostic accuracy for elevated liver enzymes were calculated.

Statistical analyses were performed using SPSS version 24 (SPSS, Inc., Chicago, IL, USA), and free statistical software EZR [27] (Jichi Medical University, Saitama, Japan). A two-sided P-value $<0.05$ was considered statistically significant.

\section{Results}

We retrospectively reviewed 37,500 Japanese women and identified 184 reproductive-aged women with PCOS, 102 of which met our inclusion criteria (Fig. 1). The calculated prevalence of elevated liver enzymes in Japanese women with PCOS was $33.3 \%$ (Fig. 1).

To find the differences between the elevated liver enzymes and control groups, anthropometric, clinical, and biochemical characteristics were compared (Table 1). The median age (29.0 years in the elevated liver enzymes group) was similar between the two groups. As expected, aspartate transaminase (AST; P $<0.001)$, ALT $(\mathrm{P}<0.001)$, alkaline phosphatase (ALP; $\mathrm{P}=$ $0.001)$, and lactate dehydrogenase (LDH; $\mathrm{P}=0.005)$ levels were significantly higher in the elevated liver enzymes group than in the control group. The correlation coefficient between ALT and AST was $0.78(\mathrm{P}<0.001)$, between ALT and ALP was $0.37(\mathrm{P}<0.01)$, and between ALT and LDH was 0.31 ( $\mathrm{P}$ $<0.01)$. Median BMI was higher $(\mathrm{P}=0.003)$ and blood glucose levels was tended to be higher $(\mathrm{P}=0.07)$ in the elevated liver enzymes group than in the control group, which are useful indexes of PCOS in clinical practice. The prevalence rate of elevated liver enzymes in PCOS patients with BMI $\geq 25 \mathrm{~kg} / \mathrm{m}^{2}$ was $58.1 \%$. LH and testosterone levels were not significantly different between the groups.

ROC analysis was conducted to assess the diagnostic value of the two models for elevated liver enzymes, with a simultaneous comparison of its components. Cutoffs for predicting elevated liver enzymes were BMI $=25.2 \mathrm{~kg} / \mathrm{m}^{2}$, blood glucose $=103 \mathrm{mg} / \mathrm{dL}$, and testosterone $=0.55 \mathrm{nmol} / \mathrm{L}$ (Table 2). In addition, AUCs derived from Models 1 (including BMI and blood glucose) and 2 (including BMI, blood glucose, and testosterone) were 0.783 (95\% confidence interval (CI), $0.664-0.901$; sensitivity $=40.0 \%$, specificity $=94.9 \%$ ) and $0.861(95 \%$ CI, $0.688-0.999$, sensitivity $=66.7 \%$, specificity $=100 \%$ ), respectively (Table 2 ). AUCs of both models were greater than the AUCs of all components and showed good or fair validity [26]. Model 2 showed the highest AUC between the two models. ROC curves of both models and their components for the prediction of elevated liver enzymes are presented in Figure 2.

\section{Discussion}

We reported a $33.3 \%$ prevalence of elevated liver enzymes in Japanese women with PCOS. The prevalence of elevated liver enzymes in PCOS women varies due to the diagnosis criteria and characteristics of cohorts [10]. An American retrospective study including 39 adolescent PCOS patients has reported that $15.4 \%$ had elevated ALT levels [28]. PCOS patients were diagnosed according to the Rotterdam criteria [29], and were of various ethnicities. On the contrary, an Italian study using the same diagnostic criteria reported elevated ALT levels (ALT > $19 \mathrm{IU} / \mathrm{L}$ ) in $57.9 \%$ of 140 overweight/ obese PCOS patients [14] which is consistent with our prevalence rate of elevated liver enzymes $(58.1 \%)$ in obese PCOS patients. Similarly, the prevalence of ultrasound-diagnosed NAFLD in PCOS varies worldwide. For example, the prevalence of PCOS with NAFLD was approximately $33 \%-39 \%$ in Chinese [30, 31], 55\% in American [32], and 73\% in Bra- 
Table 1. Anthropometric, Clinical, and Biochemical Characteristics of 102 Patients With PCOS

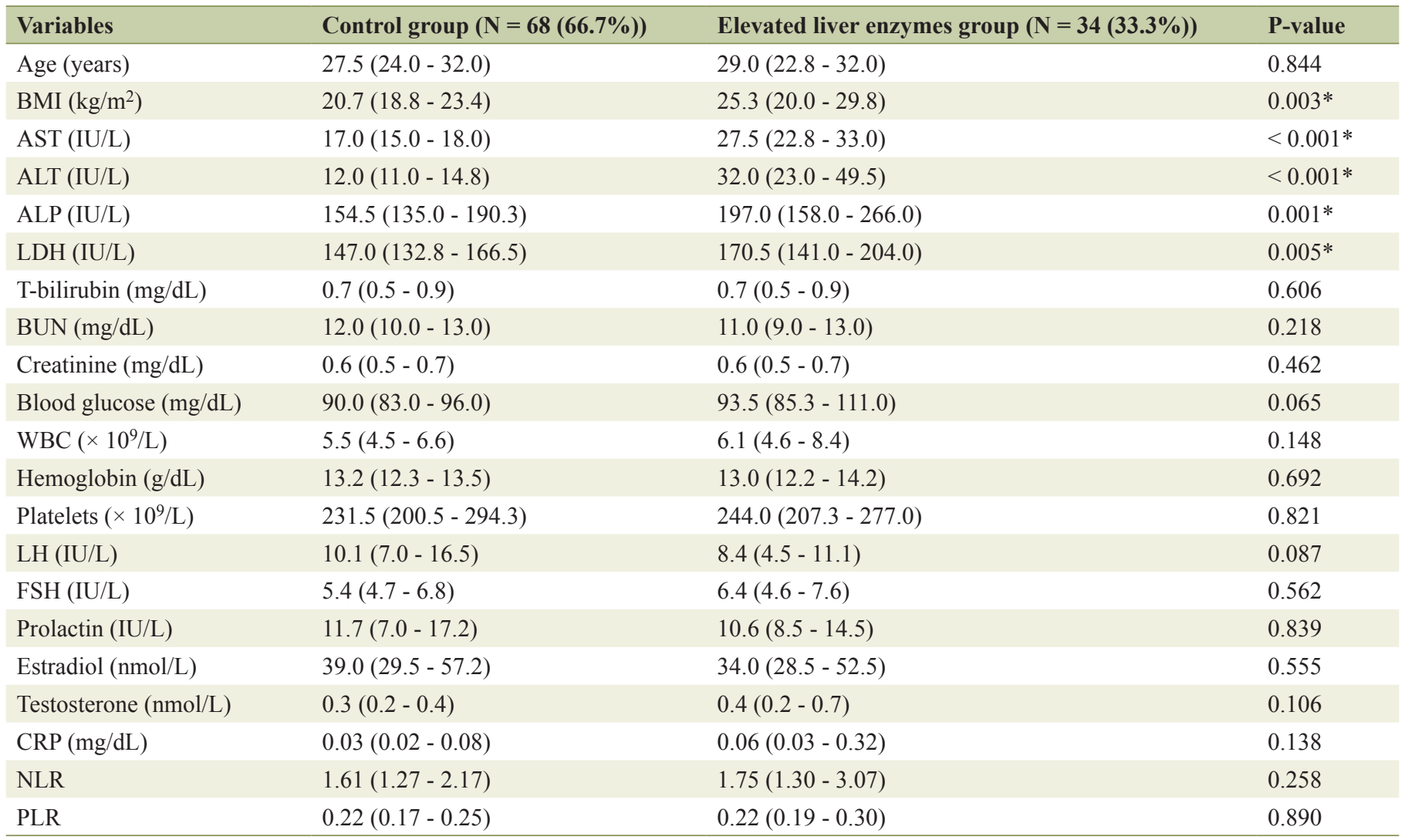

Reported anthropometric, clinical, and biochemical characteristics of 102 PCOS patients. The normality distribution of the variables was tested using the Kolmogorov-Smirnov test. Differences between the two groups were compared with Student's $t$-test for normally distributed data and the Mann-Whitney U-test for nonnormally distributed data. Continuous variables are presented as median (25 - 75 percentiles). ${ }^{*}$ Reported elevated liver enzymes group was significantly different $(P<0.05)$ than in control group. AST: aspartate aminotransferase; ALT: alanine aminotransferase; ALP: alkaline phosphatase; LDH: lactate dehydrogenase; BUN: blood urea nitrogen; WBC: white blood cell; LH: luteinizing hormone; FSH: follicle stimulation hormone; CRP: C-reactive protein; NLR: neutrophil-to-lymphocyte ratio; PLR: platelet-to-lymphocyte ratio.

zilian [33] people.

Although the Rotterdam criterion is commonly used for PCOS diagnosis in many countries, the pathology of PCOS is slightly different in Asians [34]. In this study, we used diagnostic criteria of PCOS specialized for Japanese patients [17, 18], and the prevalence was similar in Chinese patients. Unfor- tunately, obstetricians and gynecologists often do not evaluate ALT when evaluating PCOS because ALT is not a direct biomarker for PCOS. Indeed, we excluded $24.1 \%$ of the subjects because ALT was not evaluated.

A recent study has reported that triglyceride (TG)-BMI, which combines TG and blood glucose indexes with BMI, is an

Table 2. ROC Analysis of Variables in Predicting Elevated Liver Enzymes in Women With PCOS

\begin{tabular}{lllllllllll} 
Variable & Cutoff & AUC & $\mathbf{9 5 \%}$ CI & Sensitivity (\%) & Specificity (\%) & PPV (\%) & NPV (\%) & PLH & NLH & DA (\%) \\
\hline BMI & 25.2 & 0.681 & $0.563-0.798$ & 52.9 & 82.4 & 60.0 & 77.8 & 3.00 & 0.57 & 72.5 \\
Blood glucose & 103 & 0.639 & $0.483-0.794$ & 40.0 & 94.9 & 72.7 & 82.4 & 7.87 & 0.63 & 81.0 \\
Testosterone & 0.550 & 0.627 & $0.422-0.832$ & 38.5 & 87.0 & 62.5 & 71.4 & 2.95 & 0.71 & 69.4 \\
Model 1 & 7.46 & 0.783 & $0.664-0.901$ & 40.0 & 94.9 & 72.7 & 82.4 & 7.87 & 0.63 & 81.0 \\
Model 2 & 17.7 & 0.861 & $0.688-0.999$ & 66.7 & 100 & 100 & 87.0 & Inf & 0.33 & 89.7 \\
\hline
\end{tabular}

Optimal cutoffs for anthropometric, clinical and biochemical measures in 102 patients with PCOS. Sensitivity and specificity were calculated. Two models were set up for predicting elevated liver enzymes using ROC curves to determine clinical references for early diagnosis and prevention. Model 1 included BMI $\geq 25.2 \mathrm{~kg} / \mathrm{m}^{2}$ and blood glucose $\geq 103 \mathrm{mg} / \mathrm{dL}$, and Model 2 included BMI $\geq 25.2 \mathrm{~kg} / \mathrm{m}^{2}$, blood glucose $\geq 103 \mathrm{mg} / \mathrm{dL}$, and testosterone $\geq 0.550 \mathrm{nmol} / \mathrm{L}$. ROC: receiver-operating characteristics; AUC: area under the receiver-operating characteristics curve; Cl: confidence interval; PPV: positive predictive value; NPV: negative predictive value; PLH: positive likelihood ratio; NLH: negative likelihood ratio; DA: diagnostic accuracy; Inf: infinity. 


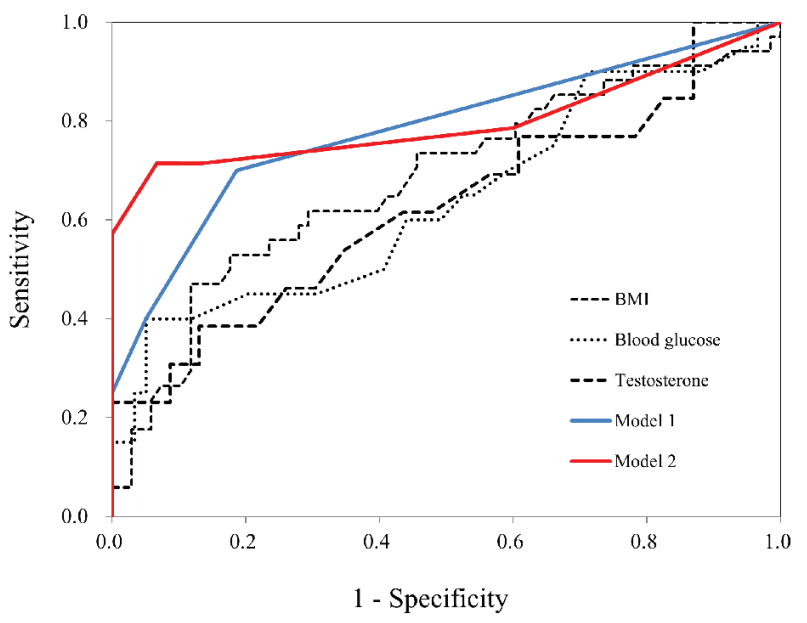

Figure 2. AUC for the prediction of elevated liver enzymes. ROC curves for BMI, blood glucose, testosterone, Model 1, and Model 2 in women with PCOS. AUC was calculated to assess overall diagnostic accuracy and to identify optimal cutoffs for risk factors for elevated liver enzymes. ROC: receiver-operating characteristics; AUC: area under the receiver-operating characteristics curve.

effective marker to detect NAFLD in nonobese patients [35]. However, the index for identifying elevated liver enzymes in PCOS women remains unknown. Therefore, we attempted ROC analysis using BMI and glucose and testosterone levels and obtained cutoffs of BMI $=25.2 \mathrm{~kg} / \mathrm{m}^{2}$, blood glucose $=$ $103 \mathrm{mg} / \mathrm{dL}$, and testosterone $=0.55 \mathrm{nmol} / \mathrm{L}$ for predicting elevated liver enzymes in PCOS women using a simple analysis. In addition, AUC using the multifactor method showed fair or good validity. To the best of our knowledge, we revealed for the first time that BMI and levels of blood glucose and testosterone can be useful indexes for predicting elevated liver enzymes in Japanese PCOS women.

A recent study has demonstrated that the appropriate cutoffs for BMI in screening for NAFLD were 24 in Chinese [30], 24.4 in Asian Indian [36], and 23.2 in Iranian [37] patients. $\mathrm{BMI}=25$ was defined as a cutoff for obesity among people in the Asia-Oceania region [38]. Our data suggested that the optimal cutoff for BMI for predicting NAFLD in Japanese PCOS women was 25.2.

Fasting plasma glucose $\geq 102 \mathrm{mg} / \mathrm{dL}$ has been regarded to be indicative of prediabetes [39]. However, cutoff levels of blood glucose in NAFLD patients are rarely considered. In this study, we showed 103 to be an appropriate cutoff for blood glucose in elevated liver enzymes women with PCOS.

Although a high LH level is considered to be related to insulin resistance, it is found in nonobese women with NAFLD, especially Asians. In addition, the frequency of insulin resistance in Asian PCOS women is lower than that in women from other countries [19]. In our study, there was no significant difference in LH, but it tended to be lower in the elevated liver enzymes group (10.1 vs. $8.4, \mathrm{P}=0.087)$. Lower $\mathrm{LH}$ and higher $\mathrm{BMI}$ in the elevated liver enzymes group are consistent with reports in Chinese women [30]. Gonadotropin-releasing hormone (GnRH), which releases FSH and LH from the pituitary gland, is inversely correlated with BMI [40]. This result seems to suggest a new mechanism of NAFLD pathogenesis without insulin resistance [41] in Japanese women with PCOS.

Hyperandrogenism is frequently observed in PCOS. Androgens, such as testosterone, dihydrotestosterone and dehydroepiandrosterone, are proapoptotic agents, and androgeninstigated apoptotic processes in the liver positively contribute to the progression of NAFLD [42]. Thus, the presence of PCOS indicates a strong proapoptotic environment, and patients with NAFLD may be susceptible to the onset of nonalcoholic steatohepatitis. In addition, PCOS patients have decreased mRNA levels of low-density lipoprotein receptors (LDLRs) in adipose tissue [42], and the activity of androgen receptor agonists attenuates the estrogen-induced upregulation of LDLRs in hepatocytes [43]. Hyperandrogenism in PCOS may have a direct inhibitory effect on LDLRs in the adipocytes and liver. Consequently, hyperandrogenism may be implicated in the presence of NAFLD in PCOS women.

Our study has some limitations. First, there may be a selective bias since the subjects had a background that required investigation of liver function; therefore, our results may represent an overestimation of the rate of elevated liver enzymes. Second, due to the cross-sectional study design, causal inferences cannot be made. To investigate the rate of elevated liver enzymes in Asians with a low prevalence of PCOS, we used data form electronic medical records of large-scale cohort, encompassing approximately 16 years. Third, although we recruited 37,500 subjects from the Department of Gynecology, the sample size was small because the prevalence of PCOS was only $4-8 \%$ in premenopausal women [5]. Fourth, PCOS is a complex of multiple diseases, and we divided PCOS patients into two groups on the sole basis of biochemical examinations in this study, leading to a heterogeneous sample. In addition, although two groups were divided on the basis of ALT, it is rare that ALT alone is high. In fact, the correlation coefficient between ALT and AST is $0.779(\mathrm{P}<0.001)$, between ALT and ALP is $0.365(\mathrm{P}<0.01)$, between ALT and LDH is $0.308(\mathrm{P}<0.01)$. These reasons could influence the anthropometric, clinical and biochemical features. Further studies are warranted for validation in another population. Fifth, we used ALT for the estimation of NAFLD. It could be insufficient to identify NAFLD with the biochemical evaluation of ALT alone. However, in this retrospective study, we included no data regarding liver biopsy or ultrasonography. In patients with PCOS, NAFLD was diagnosed by the surrogate measure of abnormal serum ALT concentrations (defined as ALT > $19 \mathrm{IU} / \mathrm{L}$ ) after excluding other secondary causes of liver disease (alcohol, virus, and medications). Further studies using more direct measures to diagnose NAFLD are required. Finally, our study did not directly investigate the degree of alcohol consumption. Therefore, we used the ICD-10 code for indicating alcohol drinkers. In addition, no patient was noted to have excessive alcohol intake in the medical records. Despite these limitations, we for the first time clarified the potential prevalence of estimated risk factors for NAFLD using elevated liver enzymes in Japanese women with PCOS. Our findings may be useful for the study of NAFLD among Japanese women with PCOS since several previous studies have indicated the elevated liver enzymes to be related to the potential presence of NAFLD. 


\section{Conclusions}

Abnormal ALT levels (>19 IU/L) accounted for approximately $33 \%$ of Japanese women with PCOS. In addition, combinations of BMI and blood glucose and testosterone levels were useful indexes for identifying elevated liver enzymes in Japanese women with PCOS. From the viewpoint of developmental origins of health and disease (DOHaD) [44, 45], it is important to provide proper nutrition education and management of body weight and blood glucose levels for prepregnancy women with PCOS to prevent lifestyle-related diseases in the next generation. Further examination of the association between PCOS and liver diseases, such as NAFLD, is required to validate these findings.

\section{Acknowledgments}

We are grateful to all of the subjects of the study for their cooperation. And we would like to offer our special thanks to all staffs of the Department of Obstetrics and Gynecology, Kyoto Medical Center, Japan.

\section{Conflict of Interest}

All the authors have reported no conflict of interest.

\section{Grant Support}

This work was supported by Grant-in-Aid for Scientific Research (B), Grant Number (JPI5H0Z90I) of Japan Society for the Promotion of Science (JSPS). In addition, the work was partially supported by the Japanese Council for Science, Technology and Innovation, SIP (Project ID14533567), "Technologies for creating next-generation agriculture, forestry and fisheries" (funding agency: Bio-oriented Technology Research Advancement Institution, NARO).

\section{References}

1. Marchesini G, Bugianesi E, Forlani G, Cerrelli F, Lenzi M, Manini R, Natale S, et al. Nonalcoholic fatty liver, steatohepatitis, and the metabolic syndrome. Hepatology. 2003;37(4):917-923.

2. Farrell GC, Wong VW, Chitturi S. NAFLD in Asia - as common and important as in the West. Nat Rev Gastroenterol Hepatol. 2013;10(5):307-318.

3. Younossi ZM, Koenig AB, Abdelatif D, Fazel Y, Henry L, Wymer M. Global epidemiology of nonalcoholic fatty liver disease-Meta-analytic assessment of prevalence, incidence, and outcomes. Hepatology. 2016;64(1):73-84.

4. Hamaguchi M, Kojima T, Ohbora A, Takeda N, Fukui M, Kato T. Aging is a risk factor of nonalcoholic fatty liver disease in premenopausal women. World J Gastroenterol. 2012;18(3):237-243.
5. Azziz R, Woods KS, Reyna R, Key TJ, Knochenhauer ES, Yildiz BO. The prevalence and features of the polycystic ovary syndrome in an unselected population. J Clin Endocrinol Metab. 2004;89(6):2745-2749.

6. Brettenthaler N, De Geyter C, Huber PR, Keller U. Effect of the insulin sensitizer pioglitazone on insulin resistance, hyperandrogenism, and ovulatory dysfunction in women with polycystic ovary syndrome. J Clin Endocrinol Metab. 2004;89(8):3835-3840.

7. Jamil AS, Alalaf SK, Al-Tawil NG, Al-Shawaf T. A casecontrol observational study of insulin resistance and metabolic syndrome among the four phenotypes of polycystic ovary syndrome based on Rotterdam criteria. Reprod Health. 2015;12:7.

8. Carmina E, Lobo RA. Polycystic ovary syndrome (PCOS): arguably the most common endocrinopathy is associated with significant morbidity in women. J Clin Endocrinol Metab. 1999;84(6):1897-1899.

9. Ramezani-Binabaj M, Motalebi M, Karimi-Sari H, Rezaee-Zavareh MS, Alavian SM. Are women with polycystic ovarian syndrome at a high risk of non-alcoholic Fatty liver disease; a meta-analysis. Hepat Mon. 2014;14(11):e23235.

10. Vassilatou E. Nonalcoholic fatty liver disease and polycystic ovary syndrome. World J Gastroenterol. 2014;20(26):8351-8363.

11. Zhang J, Hu J, Zhang C, Jiao Y, Kong X, Wang W. Analyses of risk factors for polycystic ovary syndrome complicated with non-alcoholic fatty liver disease. Exp Ther Med. 2018;15(5):4259-4264.

12. Prati D, Taioli E, Zanella A, Della Torre E, Butelli S, Del Vecchio E, Vianello L, et al. Updated definitions of healthy ranges for serum alanine aminotransferase levels. Ann Intern Med. 2002;137(1):1-10.

13. Targher G, Solagna E, Tosi F, Castello R, Spiazzi G, Zoppini G, Muggeo M, et al. Abnormal serum alanine aminotransferase levels are associated with impaired insulin sensitivity in young women with polycystic ovary syndrome. J Endocrinol Invest. 2009;32(8):695-700.

14. Gangale MF, Miele L, Lanzone A, Sagnella F, Martinez D, Tropea A, Moro F, et al. Long-term metformin treatment is able to reduce the prevalence of metabolic syndrome and its hepatic involvement in young hyperinsulinaemic overweight patients with polycystic ovarian syndrome. Clin Endocrinol (Oxf). 2011;75(4):520-527.

15. Miyake T, Kumagi T, Hirooka M, Koizumi M, Furukawa S, Ueda T, Tokumoto Y, et al. Metabolic markers and ALT cutoff level for diagnosing nonalcoholic fatty liver disease: a community-based cross-sectional study. J Gastroenterol. 2012;47(6):696-703.

16. Kwo PY, Cohen SM, Lim JK. ACG Clinical Guideline: Evaluation of Abnormal Liver Chemistries. Am J Gastroenterol. 2017;112(1):18-35.

17. The Committee for Reproductive and Endocrine in Japan Society of Obstetrics and Gynecology. Annual report (1991-1992) for the determination of diagnostic criteria for polycystic ovary syndrome. Acta Obstet Gynaecol Jpn. 1993;45:1359-1367 (In Japanese).

18. The Committee for Reproductive and Endocrine in Ja- 
pan Society of Obstetrics and Gynecology. Annual report (2005-2006) for the determination of diagnostic criteria for polycystic ovary syndrome. Acta Obstet Gynaecol Jpn. 2007;59:868-886 (In Japanese).

19. Kubota T. Update in polycystic ovary syndrome: new criteria of diagnosis and treatment in Japan. Reprod Med Biol. 2013;12(3):71-77.

20. Sunbul M, Gerin F, Durmus E, Kivrak T, Sari I, Tigen K, Cincin A. Neutrophil to lymphocyte and platelet to lymphocyte ratio in patients with dipper versus non-dipper hypertension. Clin Exp Hypertens. 2014;36(4):217-221.

21. Ratziu V, Charlotte F, Heurtier A, Gombert S, Giral P, Bruckert E, Grimaldi A, et al. Sampling variability of liver biopsy in nonalcoholic fatty liver disease. Gastroenterology. 2005;128(7):1898-1906.

22. Wang J, Xu C, Xun Y, Lu Z, Shi J, Yu C, Li Y. ZJU index: a novel model for predicting nonalcoholic fatty liver disease in a Chinese population. Sci Rep. 2015;5:16494.

23. Westerbacka J, Corner A, Tiikkainen M, Tamminen M, Vehkavaara S, Hakkinen AM, Fredriksson J, et al. Women and men have similar amounts of liver and intra-abdominal fat, despite more subcutaneous fat in women: implications for sex differences in markers of cardiovascular risk. Diabetologia. 2004;47(8):1360-1369.

24. Chang Y, Ryu S, Sung E, Jang Y. Higher concentrations of alanine aminotransferase within the reference interval predict nonalcoholic fatty liver disease. Clin Chem. 2007;53(4):686-692.

25. Zhou Z, Li R, Qiao J. Androgen profile in Chinese women with polycystic ovary syndrome in their reproductive years. Reprod Biomed Online. 2017;35(3):331-339.

26. Metz CE. Basic principles of ROC analysis. Semin Nucl Med. 1978;8(4):283-298.

27. Kanda Y. Investigation of the freely available easy-touse software 'EZR' for medical statistics. Bone Marrow Transplant. 2013;48(3):452-458.

28. Barfield E, Liu YH, Kessler M, Pawelczak M, David R, Shah B. The prevalence of abnormal liver enzymes and metabolic syndrome in obese adolescent females with polycystic ovary syndrome. J Pediatr Adolesc Gynecol. 2009;22(5):318-322.

29. Rotterdam EA-SPcwg. Revised 2003 consensus on diagnostic criteria and long-term health risks related to polycystic ovary syndrome (PCOS). Hum Reprod. 2004;19(1):41-47.

30. Qu Z, Zhu Y, Jiang J, Shi Y, Chen Z. The clinical characteristics and etiological study of nonalcoholic fatty liver disease in Chinese women with PCOS. Iran J Reprod Med. 2013;11(9):725-732.

31. Ma RC, Liu KH, Lam PM, Cheung LP, Tam WH, Ko GT, Chan $\mathrm{MH}$, et al. Sonographic measurement of mesenteric fat predicts presence of fatty liver among subjects with polycystic ovary syndrome. J Clin Endocrinol Metab. 2011;96(3):799-807.

32. Gambarin-Gelwan M, Kinkhabwala SV, Schiano TD, Bodian C, Yeh HC, Futterweit W. Prevalence of nonalcoholic fatty liver disease in women with polycystic ovary syndrome. Clin Gastroenterol Hepatol. 2007;5(4):496501.

33. Zueff LF, Martins WP, Vieira CS, Ferriani RA. Ultrasonographic and laboratory markers of metabolic and cardiovascular disease risk in obese women with polycystic ovary syndrome. Ultrasound Obstet Gynecol. 2012;39(3):341-347.

34. Huang Z, Yong EL. Ethnic differences: Is there an Asian phenotype for polycystic ovarian syndrome? Best Pract Res Clin Obstet Gynaecol. 2016;37:46-55.

35. Zhang S, Du T, Li M, Jia J, Lu H, Lin X, Yu X. Triglyceride glucose-body mass index is effective in identifying nonalcoholic fatty liver disease in nonobese subjects. Medicine (Baltimore). 2017;96(22):e7041.

36. Singh A, Parida S, Narayan J, Nath P, Padhi PK, Pati GK, Parida PK, et al. Simple anthropometric indices are useful for predicting Non-alcoholic Fatty Liver Disease [NAFLD] in Asian Indians. J Clin Exp Hepatol. 2017;7(4):310-315.

37. Naderian M, Kolahdoozan S, Sharifi AS, Garmaroudi G, Yaseri M, Poustchi H, Sohrabpour AA. Assessment of Lean Patients with Non-alcoholic Fatty Liver Disease in a Middle Income Country; Prevalence and Its Association with Metabolic Disorders: A Cross-sectional Study. Arch Iran Med. 2017;20(4):211-217.

38. World Health Organization. The Asia-Pacific perspective: Redefining obesity and its treatment. Melbourne: International Diabetes Institute. 2000;11-12.

39. Gomyo M, Sakane N, Kamae I, Sato S, Suzuki K, Tominaga M, Kawazu S, et al. Effects of sex, age and BMI on screening tests for impaired glucose tolerance. Diabetes Res Clin Pract. 2004;64(2):129-136.

40. Pagan YL, Srouji SS, Jimenez Y, Emerson A, Gill S, Hall JE. Inverse relationship between luteinizing hormone and body mass index in polycystic ovarian syndrome: investigation of hypothalamic and pituitary contributions. J Clin Endocrinol Metab. 2006;91(4):1309-1316.

41. Petaja EM, Yki-Jarvinen H. Definitions of normal liver fat and the association of insulin sensitivity with acquired and genetic NAFLD-A systematic review. Int J Mol Sci. 2016;17(5):633.

42. Baranova A, Tran TP, Afendy A, Wang L, Shamsaddini A, Mehta R, Chandhoke V, et al. Molecular signature of adipose tissue in patients with both non-alcoholic fatty liver disease (NAFLD) and polycystic ovarian syndrome (PCOS). J Transl Med. 2013;11:133.

43. Croston GE, Milan LB, Marschke KB, Reichman M, Briggs MR. Androgen receptor-mediated antagonism of estrogen-dependent low density lipoprotein receptor transcription in cultured hepatocytes. Endocrinology. 1997;138(9):3779-3786.

44. Gluckman PD, Hanson MA. Developmental origins of disease paradigm: a mechanistic and evolutionary perspective. Pediatr Res. 2004;56(3):311-317.

45. Hanson MA, Gluckman PD. Developmental origins of health and disease: new insights. Basic Clin Pharmacol Toxicol. 2008;102(2):90-93. 